\title{
Jornada sobre modelos y estrategias en salud mental
}

\author{
1. María Alonso Suárez \\ Resumen
}

En la inauguración, Alberto Fernández Liria, Presidente de la AEN, destacó la existencia actual de un consenso entre las CC.AA., plasmado en la Estrategia en Salud Mental del Sistema Nacional de Salud, acerca de la necesidad de un modelo comunitario para el que existe un conocido soporte empírico. Y exponía la situación de Madrid, donde en este momento de consenso, los poderes políticos y las circunstancias económicas promueven estrategias y modelos que llevan a la incertidumbre, por no saber si este sistema de recursos en red va a poder seguir funcionando. A continuación. Antonio Burgueño, Director General de Hospitales de Madrid, se refirió a la Jornada como un foro altamente oportuno ya que en este momento se ha propuesto hacer una revisión de nuestro sistema de Salud Mental, enmarcado en un Plan que toca revisar. Animó a los presentes a que se le hicieran llegar las conclusiones y comentó su intención de desarrollar una herramienta útil a la CAM. Pablo Rivero, Director General de Calidad y Cohesión del Sistema Nacional de Salud, expuso la necesidad de defender los avances logrados en el estado de bienestar, el SNS, y seguir avanzando sin caer en la autocomplacencia, en unos momentos de incertidumbre internacional. Destacó la importancia de haber desarrollado de manera cooperativa la Estrategia en Salud Mental del Sistema Nacional de Salud y la necesidad de que este consenso llegue a dar herramientas a los profesionales y ciudadanos.

Luego se desarrollaron las conferencias y mesas redondas, destacando la inaugural de Benedetto Sarraceno, de la OMS, que en La organización de los servicios de salud mental: perspectiva de la OMS resumió en primer lugar la evolución del abordaje de la OMS del tema de la salud mental. De una primera etapa, el Informe Mundial de la Salud Mental, 2001, destacó como palabra clave la carga que la enfermedad supone (años de vida perdidos, discapacidad, etc.) exponiendo cómo ésta es un problema grande y un problema de todos. La palabra clave de una etapa posterior de enfoque en la OMS (2004-2006) fue brecha, exponiendo la brecha que existe entre ser o no atendido con calidad: no hay suficientes recursos en muchos países, donde los hay están mal colocados (en hospitales psiquiátricos), no hay acceso a psicotrópicos en Atención Primaria y el 85\% del presupuesto se gasta en camas frente a recursos en la comunidad. Y expuso que esta brecha no es un problema «sólo en África», sino que por ejemplo el 35\% 
de los pacientes con trastornos depresivos en EE.UU. no están atendidos. La brecha está en todas partes. De la tercera etapa destacó como palabra clave las barreras: ¿Por qué existen esas barreras si sabemos que la carga es grande, que hay intervenciones efectivas, que hay tratamientos adecuados? Se refirió al estigma como una de estas barreras pero destacó que no se destina presupuesto por parte de los políticos a salud mental: porque el mensaje de los profesionales y afectados no es claro ni está consensuado.

Sin embargo, Saraceno expuso que desde la OMS existe un consenso basado en estudios científicos, de calidad, de satisfacción, en algunos aspectos: 1) Comparado con hace 50 ó 100 años, la medicina tiene más enfermos crónicos con necesidad de cuidados crónicos para los que no puede seguirse un modelo de cirujano. 2) El 85\% de los días de un enfermo crónico los pasa fuera del hospital, pero el sistema de salud sigue siendo concebido alrededor de dos ejes: a corto plazo y alrededor del proveedor (las reglas del sistema). 3) Hay que repensar el sistema de salud con esta dimensión temporal: lo crónico. 4) ¿Cómo puede ser que el $85 \%$ del presupuesto se vaya a los hospitales donde los pacientes sólo son atendidos el 15\% del tiempo? 5) En el modelo la palabra cama no debe ser clave sino la palabra plazas/oportunidades que da el servicio. ¿Cuántas de ellas tengo en mi servicio? La cama es un accidente. En vez de clínica, de estar inclinados sobre el paciente como si estuviera tumbado, necesitamos «esquizofrénicos de pie». 6) Lo comunitario en las grandes ciudades cada vez tiene menos significado; y lo comunitario no es llevar los recursos humanos del hospital a una dirección postal donde atiende ambulatoriamente un psiquiatra. 7) Es importante revisar la relación con atención primaria. 8) Es necesario un modelo con servicios que atiendan no solo a la enfermedad, sino también al daño, a la discapacidad y al handicap. La rehabilitación es una parte fundamental para la enfermedad de larga duración, para recuperar capacidades, para la reconstrucción de la ciudadanía. 9) La rehabilitación psicosocial necesita recursos, planes, tecnología, y debe ser parte de un programa global. Hay consenso en que forma parte de nuestra responsabilidad el trabajo de coordinación con los recursos sociales. 10) Es importante hablar de valores que deben transformarse en recursos, tomarse en serio el modelo biopsicosocial. Hoy nadie defendería que la enfermedad mental es sólo biológica, o psicológica, o social; no hay debate en esto. Pero, ¿cómo están organizados los servicios? Da igual que los profesionales tengan la convicción biopsicosocial si el servicio está organizado para dar una sola parte. $\mathrm{Y}$ actualmente se ha ido evolucionando hacia servicios que son bio y punto. $\mathrm{Y}$ en esto tiene mucho peso la influencia de la industria farmacéutica. 11) La «integración» debería ser la palabra clave actualmente. Y describió cómo los manicomios han ido desapareciendo a favor de instituciones sociales llevadas por empresas privadas, en ocasiones asilos con otra cara. En otras oca- 
DEBATES E INFORMES

siones disponiendo de una rehabilitación fragmentada, en la que al enfermo se le «manda a hacer rehabilitación» como quien pide una radiografía, al margen de un programa terapéutico. Puso como ejemplo la situación de Holanda, donde el hospital es lo principal y la rehabilitación psicosocial es un paquete de programas poco financiados y con profesionales con baja cualificación.

Concluyó que actualmente hay una involución del papel de la psiquiatría en la sociedad, y que estamos reanudando nudos que se soltaron tiempo atrás, que esto depende de muchos factores, pero entre otros, el nivel de miedo que existe en la sociedad. Explicó que actualmente Europa ya no es más una referencia en modelos y estrategias en Salud Mental, y que desde la OMS se siguen con interés desarrollos en Brasil, Chile, Sri Lanka, algunos estados de la India. Y por último recordó que, hoy día, la capacidad de evaluación de los resultados de los modelos es mucho mayor y que existe amplia evidencia para que el mensaje de la OMS sea: a) Menos camas y más servicios comunitarios; b) Camas en los hospitales generales; c) Tratamientos psiscosociales; d) Participación de los ciudadanos. Se abrió a continuación un debate en el que surgieron reflexiones que ahondaron en la idea de la importancia de los servicios integrados y la continuidad de cuidados, la responsabilidad de evitar una nueva generación de crónicos, y cómo recuperar el papel de la psiquiatría y la salud mental a través de conocimiento científico tanto de los modelos efectivos de atención como de los determinantes sociales de la salud (Informe de Naciones Unidas).

Las siguientes mesas redondas (en la web de la AMSM hay algunas de las presentaciones) profundizaron en muchos de los planteamientos esbozados por Saraceno. Por mencionar algunas de las aportaciones más destacadas: Pedro Cuadrado, presidente de la AMSM, que coordinaba la primera mesa redonda (Modelos y estrategias en salud mental), destacó la pérdida del papel de lo público y cómo, habiendo un consenso en el modelo, los grupos que manejan el presupuesto ponen en peligro el modelo volviendo a modelos neo-institucionalizadores. Manuel Gómez Beneyto, coordinador científico del la Estrategia de Salud Mental del Sistema Nacional de Salud (La perspectiva europea), expuso cómo en Europa, y dentro de España, existen diferencias tanto en el ritmo como el grado de des-institucionalización y desarrollo del modelo comunitario. Advirtió de los riesgos de la mayor especialización de la atención en salud mental en unidades específicas creadas por grupos de presión o por la fragmentación de la red de atención. También expuso la tendencia a la participación del sector privado, la ausencia de transparencia para poder estimar el gasto y el dato de que en general es pequeña la proporción que se dedica a salud mental de lo total invertido en sanidad si lo comparamos con la carga de la enfermedad. En cuanto a las directivas europeas, expuso las regulaciones que existen actualmente aunque resaltando que se han ido descafeinando, pasando por ejemplo de una 
«estrategia europea»a un «pacto europeo». Según Gómez Beneyto, el cambio podría estar en el papel de los usuarios si reivindican unos servicios que atiendan a sus necesidades en vez de a las del propio servicio. Y coincidió con Saraceno en que a la hora de buscar modelos quizá haya que fijarse en otro sitio y no mirar hacia Europa.

Ricardo Guinea, Delegado de Asuntos Internacionales de la FEARP, en su intervención (La rehabilitación psicosocial en el modelo comunitario), abundó en algunas de las ideas que se habían avanzado, como el consenso existente en torno al papel de la rehabilitación psicosocial, la identificación de ésta con los principios del modelo comunitario, la importancia de un modelo centrado en la recuperación o el dato de que en España se invierte en salud mental por debajo de la media Europea. Retomó la cuestión del consenso en el modelo en salud mental, mencionó los documentos de referencia nacional (Modelo de Atención a Personas con Enfermedad Mental Grave del IMSERSO y la Estrategia en Salud Mental del Sistema Nacional de Salud del Ministerio de Sanidad), que recogen de manera clara la pertinencia de un modelo comunitario con un papel fundamental de la rehabilitación psicosocial, en línea con el planteamiento de Fernández Liria, aunque al mismo tiempo se hacía la pregunta de Saraceno sobre si hay realmente consenso entre los profesionales. Guinea terminó comentando que parece que actualmente en vez de hablar de «completar el modelo» vamos hacia atrás y habría que hablar de la sostenibilidad del modelo.

Juan Carlos Casal, del colectivo Orate y vicepresidente de la Asociación Alonso Quijano (La participación y responsabilidad de los usuarios y usuarias con la salud), a partir del modelo social de la discapacidad, en el que ésta se entiende como una construcción social sobre la que no puede justificarse ningún tipo de discriminación ni limitación en la libertad de las personas, destacó el papel de los usuarios, que no puede ser substituido por las familias ni por los profesionales. Subrayó el derecho a que la capacidad jurídica de una persona con discapacidad no se viera limitada por ningún motivo y que pudieran darse otros apoyos que permitan a la persona tomar decisiones y gobernar su vida, así como el derecho a vivir de forma independiente y ser incluido en la comunidad. Por último, explicó los avances que se estaban dando a nivel nacional en torno a las asociaciones de usuarios. Tras estas tres intervenciones Gómez Beneyto fue preguntado por las consecuencias en el funcionamiento de la red tras las privatizaciones en Valencia, y destacó el daño a la integración de la red puesto que durante años han estado trabajando de manera independiente. También surgió un debate acerca de la pertinencia de que asociaciones de familiares presten servicios.

La segunda mesa, En torno a la aplicación de los modelos, coordinada por Itzhak Levav, Asesor de Salud Mental, Ministerio de Salud de Israel, comenzó con la intervención de Manuel Desviat, de la Plataforma para la Defensa de la 
DEBATES E INFORMES

Sanidad Pública, cuya presentación (Éxitos y fracasos, en torno a las Experiencias), tuvo un enfoque más micro que las anteriores, y se refirió a cuestiones acerca de si en los equipos se trabaja desde los valores y metodología del modelo, así como a la situación actual en Madrid. Se preguntaba si en la práctica de los equipos ha fraguado la práctica comunitaria y solidaria, afirmando que son las micro-experiencias aplicadas las que dan estabilidad al modelo. En su opinión, actualmente estamos frente a una crisis de valores en la que «vale todo». En cuanto a la situación de Madrid, expuso cómo actualmente Atención Primaria está estrangulada presupuestariamente, que el peso del hospital está avanzando. Se preguntaba dónde va a quedar el concepto de área en el nuevo marco de competencia libre entre hospitales. Afirmó que con los futuros planes hay programas que difícilmente se pueden llevar a cabo, como el de continuidad de cuidados.

Francisco Chicharro, director médico del Hospital Psiquiátrico de Zamudio, quien habló de Modelos, estrategias y la gestión clínica, comenzó su intervención identificándose con los profesionales formados en Leganés, y con una visión pesimista de la situación actual al observar cómo una red que ha costado tanto esfuerzo crear se puede desmantelar en pocos días con las medidas que se han tomado. Retomó alguna de las ideas que ya habían surgido en intervenciones previas, como la importancia de que las asociaciones recuperen el papel que han tenido en el pasado, el fundamental papel de los usuarios, la necesidad de distinguir entre asociaciones de usuarios y de familias, cuestionando el papel de las últimas como proveedoras de servicios. Una vez más destacó que existen suficientes evidencias como para seguir defendiendo el modelo comunitario. A partir de su experiencia en el Hospital Psiquiátrico de Zamudio expuso cómo un modelo de gestión puede servir para una cosa o todo lo contrario. Es decir, cómo puede darse un servicio de calidad y bajo un modelo comunitario desde distintos modelos de gestión. Terminó cuestionando que esto fuera posible con las iniciativas que se están tomando en Sanidad en la Comunidad de Madrid.

En torno a La fragilidad teórica en (tiempos de la) evidencia giró la intervención de Alberto Fernández Liria, Presidente de la AEN. Empezó exponiendo el «giro de los 80» en el panorama internacional tanto lo político, económico, asistencial, teórico, terapéutico, en la época de Reagan, Thatcher, con un giro de «la solidaridad al mercado», una relación con el cliente del servicio en vez de con el paciente y unos modelos de enfermedad reduccionistas con búsqueda de aplicaciones del tipo «a una enfermedad según el DSM, un tratamiento». A continuación se refirió al panorama mundial actual en sus distintas vertientes como volviendo a la solidaridad, en una crisis del libre mercado, una vuelta a la psiquiatría centrada en la persona, una neurobiología de lo relacio- 
nal, y, sin embargo, una situación en Madrid que se parece más a lo que ocurría en el mundo en los 80 , con sus fracasos, que a lo que sucede actualmente en el resto del mundo.

El debate que surgió tras esta mesa completó el objetivo de estas Jornadas: hacer un diagnóstico de la situación de la salud mental en la Comunidad de Madrid tras los cambios iniciados en Sanidad y ante la perspectiva de un próximo Plan de Salud Mental. Durante la Jornada hubo consenso en el diagnóstico de la situación en Madrid como un panorama que pone en riesgo la sostenibilidad del modelo comunitario, para el que también se destacó el consenso en torno a su efectividad en la comunidad científica y en las recomendaciones de la OMS. La reflexión llevó a cierta autocrítica por parte de los profesionales al no haber sido capaces hasta el momento de transmitir el mensaje a la población y a los políticos. Así, los representantes de las asociaciones de profesionales organizadoras de la Jornada renovaron su compromiso para incrementar las acciones de divulgación a la población, de coordinación con la Administración, de formación de los profesionales y de recogida de datos a través del observatorio de salud mental de la evolución del mapa de servicios y el modelo implementado. Clausuró la Jornada Itzhak Levav, Asesor del Ministerio de Salud de Israel.

\section{Itzhak Levav}

Claroscuros en la reforma psiquiátrica

La mayoría de las personas, si se les diera la opción, preferirían vivir en una sociedad justa y humana. Hacerlo refuerza su propia humanidad y crea confianza en el entorno. Es verdad que la Reforma Psiquiátrica (RP) reconoce la necesidad de desarrollar una acción técnica-científica de probada efectividad. Pero también es cierto que esa dimensión solo sería insuficiente para promoverla como modelo más apropiado de atención; para sostenerla, a pesar de los obstáculos; y para imprimirle el entusiasmo que una difícil tarea demanda a diario. Por eso cabe reconocer otro componente, no menos esencial. Este se refiere al afán de eliminar la distinción entre «ser como uno» y «ser el otro» en cuanto a la condición humana. Quien sufre no se convierte en «otro» por su sufrimiento. Es así que la RP postula el reconocimiento de los inalienables derechos humanos de las personas con trastornos mentales, y con los principios de la democracia -sistema que reconoce la total igualdad entre las personas-. Probablemente son estos aspectos del discurso y praxis de la RP lo que ofusca más a aquellos para quienes esos principios se tambalean, de manera abierta o disimulada. 
DEBATES E INFORMES

Juzguen ustedes: En América Latina, con el colapso de los regímenes militares y la recuperación de la democracia, surgen en algunos países o renacen en otros los movimientos de reforma. Naturalmente, el cambio operado en América Latina no es aún completo ni aceptado universalmente. Las demoras se explican, en parte, por la insuficiencia científico-técnica pero, seguramente y en igual medida, por oposiciones ideológicas. No por nuevas evidencias científicas contrarias a las que dan bases racionales a la RP sino por oposiciones ideológicas. Éstas se nutren en obstinadas convicciones de que el «otro» cesó de ser «como uno» en virtud de un trastorno psiquiátrico. De ahí a su exclusión social, apenas media un paso. ¡Qué absurdo! Para distintos sectores, sin exceptuar los profesionales, las ideas y actitudes autoritarias no desaparecieron con el final formal de los regímenes autoritarios; ambas permanecen en la mente o en la conducta y se infiltran en las gestiones de los partidos políticos y sus plataformas. Los que sostienen la RP deben estar siempre alerta frente a esta oposición. No cabe otra alternativa que concebir e impulsar las estrategias apropiadas para hacerles frente. Es la promoción de los derechos humanos el primer claro de los claroscuros de la RP. Particularmente en cuanto a la RP, es sinónimo de atención en la comunidad.

Probablemente conocen la Declaración de Caracas, adoptada en 1990 en la capital de Venezuela. En este manifiesto, 11 países latinoamericanos proclamaron su decisión de reestructurar sus servicios de salud mental tal como lo postula la RP. Con el tiempo todos los países se plegaron de manera oficial por medio de dos resoluciones adoptadas en el seno del Consejo Directivo de OPS. Quiero citar aquí a Javier Vásquez, abogado de la OPS. Él dice que «Si bien la Declaración de Caracas no se refirió explícitamente a «vivir en la comunidad» como un derecho per se, la salvaguarda de ese derecho la garantizan las orientaciones que proclama destinados a permitir que las personas con discapacidad mental vivan y sean atendidas en la comunidad». En efecto, la organización de servicios comunitarios hace posible que las personas afectadas por trastornos psiquiátricos puedan disfrutar de la libertad personal y, por consiguiente, puedan llegar a ejercer los derechos civiles, políticos, económicos, sociales y culturales y otras libertades fundamentales que les corresponde. Igualmente, la Declaración explícitamente propuso desarrollar en los países programas orientados a preservar los derechos humanos de estas personas, de acuerdo con las legislaciones nacionales y los compromisos internacionales en la materia. Sigue diciendo Vásquez que «los instrumentos de derechos humanos (tratados y recomendaciones regionales e internacionales), consagran los estándares mínimos que salvaguardan los derechos de las personas con discapacidades mentales, en cuanto constituyen un grupo que requiere protección debido a su condición de vulnerabilidad». El derecho internacional ha instituido, en relación con los derechos humanos, la protección de todas las personas sin distinción 
de su pertenencia étnica, color, edad, género, idioma, religión, afiliación política, origen nacional, extracción social, posición económica o cualquier otra característica. Consecuentemente, las personas con discapacidad mental se encuentran protegidas por los instrumentos generales adoptados internacionalmente para la defensa de los Derechos Humanos, tales como la Declaración Universal de Derechos Humanos y otros. Corresponde mencionar una vez más que, tal como lo recomendara la Declaración de Caracas, el derecho a vivir en la comunidad sólo puede ejercerse cuando los países establecen servicios comunitarios de salud mental donde las personas con discapacidad mental pueden gozar de todos los derechos civiles.

Otro claro, muy próximo al anterior. La RP sostiene el valor de la democracia. Habiendo sido definida la acción terapéutica y las intervenciones a nivel comunitario como acciones conjuntas y complementarias entre dos partes con igualdad de derechos y con obligaciones recíprocas; nada más natural que ambas partes la compartan en un diálogo continuo que nace en la determinación de las prioridades de la investigación, continúa con la programación de las acciones y concluye con la evaluación de las mismas. Este diálogo -no idéntico, pero sí complementario- se hace más patente en la atención comunitaria de la salud mental y menos, o ninguno, en la hospitalocéntrica o manicomial. En el hospital psiquiátrico, que la Reforma Psiquiátrica busca superar, la atención tiene características restrictivas. El profesional no sólo posee autoridad sino que sólo él detenta el poder, y en más de una ocasión lo instrumenta autoritariamente. Es decir, hay una relación asimétrica no democrática, no igualitaria, entre los proveedores de atención y los que la necesitan, incluidas las familias. El valor de las relaciones democráticas en el interior del sistema de salud mental constituye una de las recomendaciones de las diez que la OMS hizo en 2001, el «año de la salud mental». La OMS, a fin de impulsar el progreso en la atención psiquiátrica, recomienda involucrar a las comunidades, las familias y los usuarios; todas estas partes deben participar en la formulación y la adopción de las políticas, los programas y los servicios. El resultado esperado es desarrollar servicios más acordes con las necesidades tratadas y no tratadas de la población, y facilitar su mejor utilización. Con otras palabras: las intervenciones, comunitarias y clínicas, están de esa manera en mejores condiciones de responder a las necesidades que determinan la edad, el género, la cultura, las condiciones sociales, y la situación económica de la población por la cual los profesionales asumen responsabilidad.

Nuevamente, sin involucrar a todas las partes, la Reforma Psiquiátrica carece de bases democráticas. Ese involucramiento se extiende, amén de los aspectos antes aludidos, al proceso de capacitación. De esta manera se asegura que la capacitación cumple con su raison d'être, el sentido para la cual la capacitación exis- 
DEBATES E INFORMES

te. La Universidad debe reconocer este principio. El respeto a los derechos humanos, vivir en comunidad, y la participación comunitaria, son condiciones de la RP. Sin ellos es difícil hablar de RP, a menos que se la entienda tan solo como el mero traslado de la atención del manicomio a la comunidad. Lo que, me atrevería a decir, se trataría de un simple traslado del manicomio de dentro hacia afuera. Y algo más: la atención comunitaria tiene más posibilidades de permitir el acceso a los servicios. Para la abogada Sara Rosenbaum, en el simposio sobre cobertura para todos los norteamericanos (publicado en el New England Journal of Medicine del pasado mes de agosto), el acceso a la atención es el tema ético más esencial. Este componente ético, sólo es posible si los servicios se encuentran donde las poblaciones moran.

Habiendo visto tres claros es tiempo de mirarnos al espejo y reconocer los oscuros. Probablemente hay dos oscuros que me abruman por su omisión. El primero es la casi ausencia de toda consideración de la RP en la atención de la salud mental a niños; por ejemplo en Brasil, donde la RP está en marcha ascendente y donde la pirámide de la población es muy ancha en su base. Quizás la preocupación, admisible, claro está, por las personas adultas tratadas o depositadas en los manicomios es la que, a mi juicio, ha creado este severo escotoma. Ignorar la psicopatología de los niños es ignorar que las tasas de prevalencia de los trastornos psiquiátricos llegan al 13\% en un periodo de 3 meses, según un estudio muy bien hecho por Costello y cols. en los EE. UU. Nada más errado que suponer que toda esa carga remite espontáneamente o que carece de impacto funcional. Este impacto puede afectar el desarrollo intelectual y, por consiguiente, el potencial social del niño. Pero no solo son los trastornos psiquiátricos per se a los que importa dar solución sino a las situaciones traumáticas que viven los niños en comunidad y que tienen impacto a corto e incluso a largo plazo. Nada nuevo les digo, pero el abuso físico o sexual al niño, amén de truncar un desarrollo armónico pone en movimiento un mecanismo psicobiológico que le imprime vulnerabilidad, de tal manera que incluso en la adultez son detectables trastornos psicopatológicos severos como ansiedad, trastornos del sueño y conducta antisocial.

Veamos algunos parámetros que se pueden usar para juzgar esa omisión. En un estudio realizado en Europa, apenas unos años atrás, encontramos que la oferta de recursos humanos en psiquiatría infantil era preocupante. (Para la población de hasta 15 años, la tasa de psiquiatras de niños era de 14.0 en Italia y de 14.7 en España por 100.000, obviamente insuficiente). Este estudio informa también que en 15 países de alta renta, solo en 3 la cobertura de los servicios para niños era comparable con la de los adultos. En cuanto a los servicios para el déficit intelectual, solo en 4 países eran suficientes; en 10 eran insuficientes y en 1, simplemente, no los había. En cuanto a los trastornos de la conducta, solo en uno de los 
países los servicios eran suficientes. Datos inadmisibles que ponen en evidencia una discriminación en la atención infantil que la RP no puede tolerar. Y, hete aquí, que ninguna otra forma de asistencia está mejor posicionada que la RP, para hacer frente en la comunidad a los problemas de la salud mental de los niños, en virtud de su proximidad a los servicios pediátricos, jardines de infantes y escuelas, y a las familias.

Veamos otros oscuros en este mosaico de claroscuros de la RP. La RP hubo de reestructurar la atención curativa, frecuentemente iatrogénica, manicomial, en una amplia oferta de prestaciones que dieran respuesta a la variedad de estados psicopatológicos, cambiantes a lo largo de la vida. Pero el predominio de la atención curativa ha desplazado la atención preventiva, a pesar de ser un elemento fundamental de la atención comunitaria. La OMS ha publicado un libro con evidencias de acciones probadas científicamente. Solo identificaré una, a guisa de ejemplo. Olds mostró en EE. UU., hace años, que gracias a las visitas de enfermeras durante el embarazo y post-parto (particularmente a las mujeres en condiciones de riesgo por razones psicosociales, tal como depravación económica, hábitos de salud malsanos, deficientes prácticas de manejo familiares), el niño era menos golpeado, la lactancia materna se producía por mayor tiempo, y que disminuían los riesgos psicopatológicos, como la conducta antisocial. En síntesis, una verdadera vacuna ipsicológica! Pasma observar que una acción de tal tipo, con enfermeras o con madres graduadas en un programa de capacitación, no es parte de las acciones de rutina de los servicios comunitarios orientados por la RP.

Sigamos mirándonos en el espejo. El divorcio o mejor, la ausencia de matrimonio entre la Universidad y los servicios de la RP, empobrece a ambos. Divorcio que para la Universidad, que vive en esta realidad de torre de marfil, se traduce en una capacitación ajena o, como mínimo, distante de las necesidades de las poblaciones y en una investigación que no está encaminada a solucionar los problemas que los afecta. Nada más patente que la formación de médicos en cuanto a las materias pertinentes a la salud mental. En América Latina, por ejemplo, el médico general fracasa en la atención en salud mental. Un estudio que se realizó en seis países de Centro y Sudamérica, destinado a capacitar al médico general en la atención a la depresión, se mostró que la modificación de actitudes y conocimientos no desembocaban en una práctica diferente a la de la precapacitación. A nuestro juicio, el mal aprendizaje en la Facultad de Medicina parece ser prácticamente irreversible. Y esta es una experiencia que registra abundante literatura. El problema añadido es que los servicios, siempre escasos en recursos, deben invertir enormes esfuerzos en mejorar en algo la situación en desmedro de otras actividades necesarias que nunca cuentan, como resultado, con los recursos necesarios. Y sin el involucramiento competente del médico general, la atención comunitaria -según la promueve la RP- es parcial o totalmente imposible de llevarse a cabo. 
DEBATES E INFORMES

El dedo apostrofador no puede replegarse, la Universidad es o debe ser un organismo permeable a las necesidades de los servicios.

El empobrecimiento de los servicios por esa situación es evidente. La investigación que debe nutrir las decisiones es más escasa de la que los servicios requieren. Un estudio recientemente realizado por el Global Forum for Health Research y la OMS en los países en desarrollo mostró claramente que la investigación y la atención marchan por derroteros independientes y se unen solo en algunas oportunidades. Benedetto Saraceno y su equipo lanzaron la iniciativa Investigación para el Cambio, persuadidos que con investigación el desarrollo de los servicios se acelerará. Estos requieren del apoyo de la Universidad, como dijera, y la Universidad requiere de los servicios para cumplir su función social. El matrimonio entrambas debe producirse sin más demora.

Veamos un obvio claro. Las necesidades psicopatológicas cambian a lo largo del tiempo. Hay remisiones. Hay recaídas. Hay modificaciones en virtud de la comorbilidad que puede desarrollarse. En un estudio que realizamos en Israel en el marco del World Mental Health Survey encontramos que el 30\% de los episodios depresivos estaban acompañados de otro desorden psiquiátrico, entre ellos, 29\%, con alguna categoría diagnóstica de ansiedad. Pareciera que no encontrar comorbilidad es reflejo de un examen clínico superficial y que no se mantiene a lo largo del tiempo. Sin olvidar que la comorbilidad no solo se produce con otros trastornos psiquiátricos sino también con los físicos. Problemas cardiovasculares, úlcera, reumatismo, lumbalgias, dolores crónicos, cefaleas, fueron encontrados asociados con trastornos depresivos, los resultados de las asociaciones fueron estadísticamente significativos. Entonces, ¿cómo es posible arguiir que una opción de atención monovalente puede dar respuesta adecuada a estas necesidades? Lo que me impresionó de una visita difícil de olvidar al Servicio de Leganés fue observar cómo estaba organizada la atención de manera que pudiera dar la respuesta adecuada en el momento necesario. Están errados los que se oponen a la RP y a la atención en comunidad; la atención hospitalocéntrica y biologicista en sus diferentes variantes no responde a las necesidades cambiantes y múltiples de los miembros de la comunidad que requieren atención curativa.

La mera desinstitucionalización no es equivalente a RP. Sin ella, la RP no es completa. Pero frente a estudios que solo cuentan camas como su éxito o fracaso yo me permito apoyar a Matt Muijen, Asesor en Salud Mental de la OMS/EURO, que arguye que son las condiciones reinantes de las instituciones, su ubicación y la extensión del internamiento lo que cuenta. Es difícil que alguien se atreviera hoy a criticar las camas en medicina interna en un hospital general. Tampoco entonces cabe asumir que los internamientos psiquiátricos en un hospital general implican un fracaso de la desinstitucionalización. 
Obviamente, cabe ofrecer a la RP una solución a los internamientos en las cárceles, internamientos que parecen aumentar debido a la comorbilidad con drogas. Estas críticas desde dentro son útiles, muestran las dudas que acompañan a la RP, tal como deben existir en todo movimiento que se caracteriza por ser científico. Los directivos están atentos a los costos de las acciones de salud mental. Adviertan: esos costos son inversiones. Inversiones en la salud mental de la gente, como se dice en Chile. Menos atentos están a los costos (costos, sí) de todos los que necesitan atención y no la tienen. En el estudio WMHS que España participa, es decir, que epidemiólogos españoles participan en el ESEMeD, se encontró que 1 entre 4 personas tenían necesidades de atención no satisfechas. Es verdad, que no toda persona que sufre un trastorno psiquiátrico necesita atención, pero el problema es que aun las personas con un trastorno severo no consultan. La ausencia de consulta obedece a factores objetivos, por ejemplo, la accesibilidad de los servicios; accesibilidad en sentido amplio, no solo geográfico sino de horarios y de compatibilidad cultural. Los factores subjetivos, igualmente juegan un papel, al igual que el estigma. La RP al promover la asistencia en la comunidad está en condiciones estratégicas mejores para disminuir el sufrimiento y la discapacidad que lo acompaña. Ciertamente, la atención tradicional no puede enfrentar ni los factores objetivos, su accesibilidad es limitada, ni los subjetivos. El estigma que rodea a las instituciones manicomiales ha sido sobradamente documentado.

En síntesis, los claroscuros de la RP muestran que la tarea no está acabada. Los claros requieren refuerzos y sostenibilidad; los oscuros demandan inmediata solución.

Permítanme concluir con un mensaje personal. En este acto se celebra la trayectoria de Manuel Desviat y sus colegas. Pero, Manuel Desviat no solo desarrolló la RP en Leganés. La llevó a Brasil, donde la reforzó, y a México, donde la RP diera sus pasos iniciales titubeantes, en misiones sucesivas de OPS, en el marco del movimiento de reestructuración de la atención psiquiátrica de América Latina. Su tarea fue descollante, los testimonios son de los profesionales locales y la evidencia de los resultados concretos. Querido Manuel, con pena, asombro e ira me enteré que fuiste removido de Leganés. Las razones son sinrazones, a mi juicio. La solidaridad y el apoyo de los usuarios, familias, comunidad, discípulos, pares y amigos son el mejor reconocimiento de los éxitos de tu tarea. Pero, aunque todo esto nutre, en todo momento y en época de crisis más sin duda, que no solo eso te sirva de nutriente. Que sean las acciones venideras las que te nutran porque la tarea no está terminada y la misión no ha concluido. Todos, sin excepción, todos, esperamos que continúes tu tarea y que prosigas con tu misión. $\mathrm{Y}$ a ustedes, les agradezco el honor que me han hecho al invitarme a compartir la plataforma con líderes de la RP en este país. 


\section{Manuel Desviat}

\section{Éxitos y fracasos: en torno a las experiencias}

I.- Hablar de logros y de insuficiencias, hacer balance de lo conseguido y de lo que falta, tanto en lo macro, en el escenario de las estrategias generales de la reforma, como en lo micro, en las experiencias asistenciales que constituyen el forjado de todo el proceso, significa plantearse no solo lo alcanzado, sino también preguntarse sobre su sostenibilidad.

Hay que plantearse qué de lo conseguido tiene suficiente consistencia, ha cambiado suficientemente la realidad, para que subsista en el tiempo. ¿Cuáles de los nuevos programas han invalidado a prácticas anteriores? Más aún cuando estamos en un tiempo de crisis de valores, falto de grandes teorías y principios, donde todo parece valer (para que al final valga lo que se acomoda más fácilmente a la ganancia del capital. El mercado manda).

No cabe duda de los avances alcanzados en la asistencia psiquiátrica de este país desde la transición democrática, ni del peso que en este proceso han tenido los profesionales y las asociaciones, como las que convocan este acto, en la mejora de la atención a la salud mental, y en el intento de cambiar las formas de hacer la asistencia. Tampoco de los límites que la evolución política del Estado y sus Autonomías han impuesto al desarrollo de una atención comunitaria. Pero la cuestión es, y aquí entran en juego las experiencias concretas, en un Estado donde las diferencias de desarrollo asistencial entre CC. AA., y aun dentro de éstas, es enorme; la cuestión es preguntarnos sobre el grado de implantación del modelo propuesto en la reforma sanitaria y psiquiátrica; preguntarnos, no ya desde la extensión de los nuevos recursos, desde el crecimiento de personal y servicios, sino desde la práctica de los equipos, desde los programas, desde la clínica, preguntarnos qué ha fraguado de aquella idea comunitaria y solidaria. Hasta dónde ha sido posible. Pues, de esta implantación y de esta consistencia, va a depender en buena medida la sostenibilidad de toda la Estrategia, lo que pueda mantenerse del modelo.

Más aún, si tenemos en cuenta la gran vulnerabilidad de especialidades tan ideologizadas como las de salud mental, tan sensibles a los vaivenes de los partidos políticos. Debilidad que adquiere mayor relevancia, cuando lo que se pretendía en la Reforma Sanitaria que se inicia con la LGS, era un cambio del Sistema Sanitario y de su modelo organizativo; un cambio que diera una mayor cobertura a la población, pero también OTRO tipo de asistencia, otro tipo de acercamiento al malestar psíquico; una atención a la persona que tuviera presente al sujeto y no solo a sus síntomas y aparatos; que tuviera presente su subjetividad, y por tanto, su autonomía, su dignidad y su libertad. Que tuviera presente a las colectividades y a la persona tanto en comunidad como indivi- 
dualmente. Y una reforma que además empezaba ya a contracorriente, cuando Thatcher, Regan, el Banco Mundial iniciaban su giro neoliberal, sus recortes del Estado del Bienestar.

Psiquiatría social, salud mental comunitaria, psiquiatría pública, abordaje biopsicosocial, psicopatología del cuidado... Ese era el objetivo, y es a partir de esa perspectiva desde donde creo necesario abrir una reflexión para acercarnos a la situación actual, éxitos y fracasos de la reforma. Una reflexión que debe partir de las asociaciones que defienden la salud pública; asociaciones como las convocantes de este acto, sobre todo en los lugares, en las Comunidades, donde se ve amenazada, donde el contexto político es claramente adverso, si queremos diseñar una estrategia de progreso o simple resistencia.

II.- Empezaré por ver qué ha pasado con el Área Sanitaria y la Atención Primaria, dos componentes fundamentales del diseño de la reforma sanitaria española, consideradas con razón internacionalmente lo más innovador de la transformación de la Sanidad de nuestro país. Dos componentes que inciden particularmente en la atención a la salud mental, en una estrategia de cambio que sitúa a la psiquiatría y la psicología clínica en segundo nivel, especializado, dentro de la red sanitaria del Área de Salud.

Hoy, nadie discute el desarrollo de la atención primaria, pero tampoco se duda de su estrangulamiento actual, pero cada vez hay más datos sobre sus dificultades, voces sobre su relativo fracaso, por falta de medios, de tiempo, de presupuesto. Un presupuesto cuyo porcentaje -hospital-atención primaria- atribuido sigue siendo uno de los más bajos de Europa. Algo que es más pronunciado en las CC. AA. más ricas que orientan todavía más su gasto hacía los hospitales. Son las más pobres, envejecidas, o las gobernadas por la izquierda las que favorecen la AP. Plataformas como las de diez minutos, que exigen un tiempo mínimo para cada paciente (o la pretendida absorción de la atención primaria por entidades hospitalarias privadas en los planes de la Comunidad de Madrid), señalan no el fracaso de un modelo ejemplar, sino su paulatino estrangulamiento. De sus dificultades, de su cansancio, de su hartazgo dan cuenta a diario manifestaciones y paros en todo el Estado.

Respecto del Área de Salud, elemento marco de la ordenación sanitaria de la LGS, habría que preguntarse dónde están los Consejos de Salud; ¿dónde la integración de salud pública, primaria y especializada?, ¿dónde queda el Área en un sistema regido por la competencia entre centros y la adjudicación capitativa de la financiación, en un sistema como el que se quiere o se está ya imponiendo en la Comunidad de Madrid?

III.- La psiquiatría, la salud mental se enfrentó a un doble desafío: desmontar un sistema antiguo asentado en el hospital psiquiátrico y las consultas del seguro y, a la vez, a la construcción de un nuevo modelo asistencial, de una 
nueva clínica, que superara la idea que de la psiquiatría habían trasmitido unas cátedras monocolor, que se resistían a los avances de las especialidades de la salud mental. Un desafío al que con los años y la evolución conservadora de la sociedad, se une el cada vez mayor peso de la mentalidad hospitalaria -ahora del hospital general- y la amenaza de sus consultas externas, monográficas, por patologías frente a los equipos comunitarios; o lo que es lo mismo, el mayor peso, de nuevo, con todas sus consecuencias, del Servicio de psiquiatría frente a los Servicios de salud mental.

Una muestra de las consecuencias de este desplazamiento del eje de la asistencia de la comunidad al hospital está en la manera de medir por los gerentes la actividad ambulatoria: se preocupan tan sólo de las primeras y segundas consultas, y penalizando el mayor número de estas segundas, lo que demuestra su pobre noción de la actividad psiquiátrica. La ausencia de indicadores propios de la salud mental, de guías y protocolos, de procesos consensuados de ámbito estatal, ausentes también en muchas de las CC.AA., deja inerme la salud mental en su necesaria integración en la sanidad general. O la sitúa en su concepción meramente biológica, o psiconeurofarmacológica, tan deseada por la Psiquiatría Perdida entre sus Pruebas.

En este escenario, los programas de continuidad de cuidados, las acciones de ámbito más comunitarias quedan fuera, como pueden quedar fuera en la Comunidad de Madrid los programas de rehabilitación que en su inmensa mayoría, dependen de Servicios Sociales. O los recursos residenciales, fuera de las redes de las áreas, en renovados, y poco, hospitales psiquiátricos; con camas, de nuevo, en otra Comunidad Autónoma. Con estos límites, con las agendas llenas de pacientes crónicos más o menos estabilizados, de trastornos comunes por mor de la medicalización de la vida, de la psicologización de los factores de riesgo y la psiquiatrización de las pre-enfermedades; con los agudos y crónicos desestabilizados colapsando las urgencias, con la presión de más pacientes por día de los que se puede atender adecuadamente, con la falta de una política de salud mental, con la delegación de la formación en la empresa farmacéutica, con la falta de motivación económica y profesional, con jefes de salud mental regionales sin la autoridad suficiente -lo que demuestra la poca importancia que le dan a la salud mental- para ser interlocutores capaces con las gerencias y la Administración Sanitaria; o también en la Comunidad de Madrid con la salud mental escindida en centros de salud mental y unidades hospitalarias, islas o guetos independientes cada centro; convertidas en ambulatorios o unidades de crisis, con políticas defensivas cada vez más coercitivas y biológicas; con esto -y más quizá- sobra plantearnos la construcción de una nueva clínica, de un nuevo discurso, rescatando lo hecho durante la reforma. 
Puede que en esta situación, esté de más preguntarnos, cómo se atiende hoy a los pacientes en los centros de salud mental, en los hospitales de día, en las unidades psiquiátricas de los hospitales generales, en los programas de rehabilitación. ¿Qué idea o ideas de la enfermedad mental sustentan nuestra práctica? ¿Qué papel tiene la comunidad, las familias, los ciudadanos en estos programas de salud mental? ¿Qué de la participación, de la autonomía del paciente mental? En definitiva, cuesta preguntarnos qué hay de la psiquiatría comunitaria ¿hasta dónde, o hasta cuánto, nos diferenciamos en nuestra práctica habitual de la psiquiatría tradicional, biológica, anclada en el psicotropo?

Sin embargo, creo que debemos buscar la manera de hacerlo, debemos abrir una nueva reflexión colectiva, usando como antaño las asociaciones, los márgenes del sistema, allí donde el sistema es adverso. Es una cuestión de supervivencia, de supervivencia del modelo.

Tenemos experiencias sobre la diversidad y desigualdad que han creado las transferencias y la ausencia de autoridad por parte del Ministerio de Sanidad y Consumo; experiencias que han desarrollado acciones de atención comunitaria, por ejemplo en la atención a la cronicidad, donde hemos dado grandes pasos, donde tenemos programas eficaces, más eficaces que nunca, pero flacos, en muchas ocasiones, psicopatológicamente, como diría Fernando Colina, y no siempre respetuosos con la autonomía del paciente, lejanos a las ideas de recuperación y empoderamiento en auge hoy en día.

La participación ciudadana es casi inexistente y la seducción psicofarmacológica está invadiéndolo todo, desde la psicología hasta las familias. Seducción farmacológica que trae de la mano la idea de la normalización, el chantaje de la normalización paternalista; que el otro, que el diferente deje de ser diferente, y donde los síntomas dejan de tener un sentido existencial. El pragmatismo desaloja la psicopatología, DSM en mano, y una clínica que se base en el respeto y el cuidado. Salud mental de la receta, el consejo y la conciencia de enfermedad como mito. ¡Parece como si volviéramos a la idea de la curación!

IV.- Y aquí entran en juego las experiencias: la construcción del modelo se hace en el marco de las estrategias establecidas por la Autoridad Sanitaria, pero su concreción, su desarrollo, va a estar marcado por experiencias institucionales particulares, más o menos globales. Experiencias que actúan como vanguardias en la creación, experimentación y desarrollo de nuevas prácticas, de nuevos conocimientos (rompiendo límites, creando nuevas reglas, ensayando caminos). Experiencias pioneras cuyo éxito depende de si han podido mantenerse, aún a contracorriente, el tiempo suficiente como para crear nuevas vías y demostrar su viabilidad o verificar aquello que querían demostrar. Creando y expandiendo fuera de sí mismas los profesionales que puedan continuarlas. 
Por su carácter de vanguardia estas experiencias, más visibles, mostradas por la propia administración como su obra, son procesos muy vulnerables, sobre todo porque precisan de libertad, como lo precisa todo conocimiento, para encontrar nuevos caminos y desarrollarlos.

Pero hay que tener en cuenta que la independencia, esencial en las sociedades democráticas en áreas como la Sanidad o la Universidad, es incómoda para el Poder Político, sobre todo cuando éste se rige más por intereses de partido que universales. No podemos olvidar que la reforma sanitaria, al igual que la Transición democrática fue un pacto, y en condiciones desiguales. La psiquiatría tradicional mantuvo la hegemonía en la Academia, en la Universidad. Por error de cálculo o incapacidad del movimiento progresista.

En la asistencia, a falta de una coordinación por parte del Ministerio de Sanidad, la reforma se desarrolló en las Comunidades de forma tremendamente desigual, y en muchas de ellas gracias a la buena voluntad de algunos políticos y gestores, y siempre desde el voluntarismo de los profesionales, con acciones carentes en muchos casos de la normativa legal que les apoyara, acciones comunitarias que tenían que transgredir organigramas, normativas hospitalarias, y realizar actividades no contempladas en los convenios colectivos. Creo que uno de los grandes fallos de la reforma ha sido precisamente esta falta de normativas, que el Estado no regulara unos mínimos asistenciales, tanto de indicadores, como de denominación, contenido, funciones y personal de los servicios de salud mental. Ausencia que ha propiciado grandes desigualdades y una absoluta falta de información por el Estado. Indefiniciones legales, o normas como la ley 15/1997, abren la puerta a formas de gestión y financiación que pueden acabar con el modelo de asistencia a la salud mental que contempla la LGS, o en el caso de Madrid, el mismo Plan de Salud Mental de la propia Comunidad.

V.- Volviendo al principio, quiero insistir en la idea que mueve mi intervención. Una idea quizá condicionada por el momento lleno de incertidumbres pero con algunas certezas, que suponen una gran amenaza para la asistencia psiquiátrica comunitaria allí donde prospere un sistema sanitario regido por la competencia y no por la equidad y la solidaridad. Una idea que me lleva a creer que el futuro, el futuro de la salud mental comunitaria, su posibilidad misma, está en el desarrollo de una clínica comunitaria. Desarrollo que exige el esfuerzo de las asociaciones y colectivos progresistas. Que exige construir un nuevo discurso desde nuestro trabajo cotidiano, desde los centros de trabajo asistencial. La reforma ha conseguido modificar radicalmente los espacios de tratamiento, ahora queda modificar los tratamientos. 


\section{Benedetto Saraceno}

¿Por qué urge actuar? Entrevista

A. F. L.: La OMS ha realizado, durante los años en que diriges el Departamento de Salud Mental, una incesante investigación sobre la situación de la salud mental en el mundo. ¿Podrías hablarnos de esta situación?, ¿qué perspectivas ves tanto en los países desarrollados como en los países pobres?

B. S.: Una de cada cuatro personas -el $25 \%$ de la población-, sufre en algún momento de su vida al menos un trastorno mental. Actualmente hay 450 millones de personas con trastornos mentales en países tanto desarrollados como en desarrollo. Los problemas de salud mental constituyen cinco de las diez principales causas de discapacidad en todo el mundo, lo que supone casi un tercio del total de la discapacidad mundial. Los trastornos con mayor peso son la depresión, el abuso de sustancias, la esquizofrenia y la demencia. Esta carga cobra un elevado tributo en forma de sufrimiento, discapacidad y pérdidas económicas. Aunque los trastornos mentales afectan a personas de todos los grupos sociales y países, los pobres los sufren de forma desproporcionadamente mayor. Además, dado que la longevidad se acrecienta y las poblaciones envejecen, en las próximas décadas aumentará el número de afectados por trastornos mentales, así como la carga consiguiente. En todo el mundo, numerosas personas con trastornos mentales son víctimas de discriminación por el simple motivo de padecer esos trastornos. Con frecuencia tienen dificultades para conseguir vivienda, empleo u otros servicios que forman parte de la vida normal en la sociedad, lo que a veces provoca la agudización de sus síntomas. De ahí que a menudo vacilen en recurrir a especialistas para tratar sus problemas y opten, en cambio, por sufrir solos y en silencio. La información equívoca sobre los trastornos mentales, por ejemplo, la idea de que esos enfermos suelen ser violentos o víctimas de algún tipo de maleficio, o de que esas dolencias son incurables, no hacen más que perpetuar el estigma y la discriminación. La falta de acceso a terapias eficaces y el internamiento de esas personas en centros psiquiátricos aislados, dan pábulo a ese tipo de prejuicios. En los Principios de las Naciones Unidas para la Protección de los Enfermos Mentales y para el Mejoramiento de la Atención de la Salud Mental se establece que no habrá discriminación por motivo de enfermedad mental, que todo paciente tendrá derecho a ser tratado y atendido en la comunidad en la que vive y a recibir el tratamiento menos restrictivo posible. La aplicación de esos principios, sin embargo, dista mucho de ser perfecta en buena parte del mundo.

Nuestra comprensión de los trastornos mentales y de su tratamiento progresa con rapidez. También sabemos que en la mayoría de los casos es posible tratarlos con buenos resultados. Con una terapia apropiada pueden controlarse eficazmente los síntomas de cerca del $70 \%$ de los casos de depresión, esquizofrenia y 
DEBATES E INFORMES

epilepsia; la aplicación sostenida del tratamiento reduce sustancialmente las probabilidades de recaída. La farmacoterapia resulta efectiva y además bastante barata en muchos países. La intervención psicosocial es un componente básico, y en muchos casos rentable, del tratamiento de la mayoría de los trastornos mentales y de la rehabilitación de los pacientes. Otros avances importantes en el terreno terapéutico son resultado de los movimientos de los usuarios y de defensa de los derechos humanos. Ambos movimientos han prestado especial atención a las violaciones de los derechos de las personas con enfermedades mentales y han insistido en la obligación que tienen los poderes públicos de promover y proteger los derechos e intereses de esas personas. Esos movimientos han propugnado, asimismo, la elaboración de normas de garantía de calidad y la búsqueda de alternativas al internamiento en centros psiquiátricos.

Aunque en teoría exista la posibilidad de hacer frente con éxito a los trastornos mentales, en la práctica sólo se dispensa tratamiento, aun del tipo más básico, a una pequeña minoría de quienes lo necesitan. Un reciente estudio de la OMS sobre los recursos de los países en el ámbito de la salud mental (Proyecto ATLAS, 2000-2001), ha reunido información sobre 185 países (96,9\% de los Estados Miembros) que comprenden, en conjunto, al 99,3\% de la población mundial. Los análisis de esos datos han puesto de manifiesto que: a) el $41 \%$ de los países no tiene definida una política de salud mental; b) el 25\% carece de legislación en la materia; c) el 28\% no dispone de un presupuesto independiente para la salud mental. (Entre los países que informaron tenerlo, el 36\% destina a esta área menos del $1 \%$ de su presupuesto total de salud); d) el $37 \%$ carece de establecimientos de atención comunitaria de la salud mental; e) en más del $25 \%$ de los países los centros de atención primaria no tienen acceso a medicamentos psiquiátricos esenciales; f) en más del $27 \%$ no hay ningún sistema para recoger y comunicar información relativa a la salud mental; g) alrededor del $65 \%$ de las camas destinadas a esta atención se encuentra en hospitales psiquiátricos autónomos; h) el 70\% de la población mundial dispone de menos de un psiquiatra por cada 100000 personas.

Con frecuencia, los recursos para la salud mental ofrecen un estado desolador. Los recursos y servicios con frecuencia representan una décima o incluso una centésima parte de los necesarios. Sin embargo, hay muchas diferencias entre países y no es posible tener un mapa completo de la situación: algunos tienen problemas de salud mental muy específicos (por ejemplo altas tasas de suicidio o abuso de alcohol); otros tienen sistemas de salud mental muy atrasados o, al contrario, muy desarrollados. Quizás algunos ejemplos concretos puedan ayudar. Ex Repúblicas Soviéticas como Lituania, Estonia, Latvia, Rusia misma y también Suiza, Luxemburgo, Bélgica tienen una proporción importante de suicidios. El consumo de alcohol está aumentando y se amplían las condiciones que nunca se veían antes, como el síndrome fetal de alcohólicos, quiere decir que hay chicas 
embarazadas consumidoras de alcohol de forma dramática. El aumento de los refugiados, de las emergencias naturales, de los conflictos armados, o de lo que sea, incrementa fuertemente la problemática de Salud Mental, y la carga aumenta. La respuesta es que no hay nuevas grandes experiencias innovadoras, seguimos con experiencias puntuales quizás debidas a un líder local, debidas a una contingencia, y si hacemos un mapa de las reformas innovadoras en Salud Mental, aparte de las tradicionales reformas innovadoras europeas de España, Italia, Inglaterra y en Latinoamérica, con Chile y Brasil liderando el proceso de reforma, no hay mucho más. A pesar del Informe Mundial del año 2001, de la Declaración de Caracas y de la Declaración de Helsinki, las reformas realmente innovadoras en Salud Mental siguen siendo muy pocas, y continúan al contrario las respuestas tradicionales: manicomios, o en ausencia de manicomios, en países muy pobres, el abandono.

A. F. L.: No hay muchos, ni en los países desarrollados ni en los países de bajo nivel de ingresos. ¿Habría una diferencia, en estos momentos, entre ellos si pensáramos en las posibilidades de desarrollar algo parecido a servicios de atención comunitaria?

B. S.: Hay algunos aspectos en que los países desarrollados tienen una mejor capacidad para desarrollar la Salud Mental Comunitaria y otros aspectos en que son los países subdesarrollados los que tienen mejor capacidad: por ejemplo, el rol de las cooperativas, las viviendas protegidas, han sido mucho más incorporados en las experiencias europeas que en las experiencias de los países en vías de desarrollo. Entonces, si hoy la OMS necesita a un asesor, a un consultor en estas áreas, sería seguramente un consultor europeo, pero no me cabe la mínima duda de que si por ejemplo, miramos a la relación con la Atención Primaria en muy remotas regiones de gran pobreza, los países en vías de desarrollo han tenido una capacidad de inyectar Salud Mental a nivel de Atención Primaria mucho más que nosotros los europeos. En asociaciones, por ejemplo Brasil tiene un movimiento de usuarios que tampoco vamos a imaginarlo nosotros en Europa. Entonces hoy, para la situación ideal, necesitaríamos un pedazo de Brasil, otro de Trieste, otro de España, para juntarlos. Sigo pensando, por ejemplo, que la experiencia española con todos los aspectos de la formación de cooperativas es bastante única en Europa, y que la integración público-privado, privado no con fin de lucro, privado-social digamos, ha sido una experiencia importante en España. En conclusión no veo un país liderando el proceso; quizás hace 30 años era Italia pero no hoy: no está liderando nada. Existen trayectorias innovadoras en área de derechos humanos, hay países que son muy sensibles en el área de Atención Primaria, otros países en el área de desmanicomialización: hay experiencias con redes amplias de prestaciones, pero son minoritarias. Al tiempo, crece un proceso de privatización muy fuerte que va a empeorar las cosas; es seguro que en el momento en que se fragmente la oferta de servicios entre varios actores sociales, privados, privados 
DEBATES E INFORMES

subvencionados, públicos, también el paciente se encontrará fragmentado, y creo que esto va a ser nefasto y veo el riesgo en Europa de una separación cada día mayor entre lo que es social y lo que es salud, con el sector salud que se limita a la dimensión médica (que al final es el tratamiento hospitalario y las drogas) y deja lo social bajo el control de un incierto mundo de competencias, como algo de cuarta clase, de quinta clase.

A. F. L.: Algo que tendría que ver con la calidad, casi.

B. N.: Sí, con lo de la rehabilitación de trastornos en lugares donde se pone la gente ahí escuchando una musiquita y esta es la rehabilitación. En nuestra idea, la rehabilitación era la reconstrucción de la ciudadanía, no el entretenimiento caritativo de los pacientes mentales

A. F. L.: Y ¿cómo podríamos imaginar un posible futuro, teniendo en cuenta que vivimos un contexto económico que parece que no da para grandes alegrías?

B. S.: Hay que recomenzar la noción de movimiento de abajo hacia arriba. Digo esto porque, por un momento, los reformadores han sabido controlar algunos países o provincias, o regiones o ciudades, y han tomado un liderazgo cultural. Entonces, la Salud Mental innovadora ha sido hegemónica, por lo menos su discurso, y en algunas zonas también en la práctica. Ahora no es así, y por razones que van más allá de la psiquiatría. Hay un aumento de los miedos relacionado con la seguridad en Italia y en Francia, debido a los inmigrantes, a los ilegales, y todo este discurso ahora sobre la criminalización del trastorno de personalidad, por un lado, y por otro la presión de la industria farmacéutica, que son fenómenos que han empobrecido la cultura psiquiátrica de las generaciones jóvenes de psiquiatras. Me parece que un joven psiquiatra de los años sesenta u ochenta era más holista en su visión de su profesión de lo que pueda ser hoy un psiquiatra que tenga 30 ó 35 años, y eso debido a una fuerte presión de parte de la industria que ha conquistado cabezas, mercados y al final gastos porque el gasto no es infinito: si el presupuesto se utiliza solo para comprar nuevas moléculas, al final habrá menos plata para pagar enfermeras que hagan visitas domiciliarias.

Creo que, humildemente, hay que retomar la ruta del movimiento de base, y tenemos un aliado que en los años setenta no sabíamos identificar, o sea el movimiento de los usuarios, que tiene una fuerza, una capacidad de provocación muy importante. Además, existe una menor necesidad de ideologizar lo que estamos proponiendo. Tenemos una actitud más pragmática, lo que nos facilitaría el no identificar inmediatamente el proceso de reforma con discursos políticos o partidistas y esas son nuevas herramientas: conviene retomar el movimiento de abajo hacia arriba porque no creo que el proceso de cambio pueda llegar de la OMS o de la Unión Europea o de los gobiernos, si no hay una capacidad de crear un movimiento crítico importante, entonces el futuro es retomar el espíritu de lucha que se ha perdido. 
A. F. L.: Esto casi responde a mi última pregunta. Igual hay algo más que se puede decir con respecto a lo que sucede en este momento en algunas Comunidades españolas, muy en concreto en Madrid. Una situación en la que se está produciendo una retirada de los recursos públicos del terreno de la atención sanitaria mientras que a los empresarios que hipotéticamente podrían invertir en este campo desde la Consejería de la Salud, se les ofrece un terreno en el que uno puede aprovechar las oportunidades para tener beneficios. Si tuviéramos que lidiar en un panorama configurado por este punto de partida, ¿qué lecciones de otros lugares del mundo podríamos intentar aplicar aquí?

B. S.: No tengo una respuesta. Creo que la palabra clave es equidad. Si la recomendación de la OMS es que el sistema de salud es un sistema fundamentalmente público, sin duda hay que tener en cuenta un cambio social en que deberá aumentar la interacción público-privado, desde lo privado-social sin fin de lucro a lo privado de Organizaciones No Gubernamentales, y también quizás en algunos casos a lo privado con fines de lucro. Sin embargo, el proceso de transformación de salud en negocio no va a ayudar a respetar los grandes puntos clave de la OMS, o sea la equidad, la accesibilidad y la salud para todos. Eso que tenemos que mantener como referencia ética y técnica.

A. F. L.: Sí, deberíamos poder salvaguardar eso.

M. D.: Llevas años dirigiendo la salud mental en la Organización Mundial de las Naciones Unidas, me gustaría saber qué dificultades has encontrado en tu trabajo -no digo en la asistencia, sino en la propia función-, qué oportunidades le ves al Organismo, qué posibilidades tiene de influir sobre los distintos países.

B. S.: Tengo dos respuestas. En primer lugar, seguramente la estigmatización que sufre la Salud Mental, la sufre también dentro del sistema. El prejuicio de que Salud Mental no es a fin de cuentas una gran prioridad, lo sufrimos también dentro del sistema de Naciones Unidas. Si miras los Objetivos del Milenio de Naciones Unidas, la Salud Mental no aparece, están solamente las enfermedades transmisibles. Creo que habría que pensar en la fuerte relación entre enfermedades físicas transmisibles y no transmisibles y la enfermedad mental, pues la ausencia de salud mental es un grave factor de riesgo también para la enfermedad física. El lema no health without mental health no creo que sea retórico, no hay salud sin salud mental, entonces la primera respuesta es que también hay que conducir un movimiento de promoción dentro del sistema para que la Salud Mental ocupe la plaza que tendría que ocupar.

¿Cuál es la capacidad de influencia que tiene OMS? En países de bajos ingresos tiene una influencia mayor que en países de altos ingresos: es evidente por una razón muy simple, que los países grandes industrializados y muy desarrollados tienen expertos internos. Si el Ministro de Salud de Inglaterra quiere hacer la reforma de salud mental, no necesita al Dr. Saraceno de Ginebra, tiene en 
DEBATES E INFORMES

Inglaterra bastantes instituciones competentes para que le puedan apoyar y técnicamente ayudar, eso no es sorprendente. Mientras que si Honduras quiere hacer una reforma en salud mental necesita el apoyo de organismos como la OMS. Entonces nuestra influencia disminuye al aumentar el grado de desarrollo de los países, eso es evidente. Y un elemento que no existía en el pasado: hay más competencia, hay otras agencias que tienen una influencia en Salud Mental como el Banco Mundial, que tiene expertos en Salud Mental que acompañan en préstamo al país. Sin embargo, cuando el préstamo es en salud a veces hay un componente de Salud Mental y no siempre el discurso del experto del Banco Mundial y el discurso del experto de la OMS coinciden. La OMS no tiene un liderazgo asegurado, tiene que conquistar el liderazgo moral y técnico en cada país y en cada situación.

Palabras recogidas por Alberto Fernández Liria y Manuel Desviat

* Jornada organizada conjuntamente por la AEN, FEARP y la Plataforma por la Defensa de la Salud Mental Pública. Se celebró el 30-IX-2008, en el Colegio Oficial de Médicos.

** María Alonso Suárez es Secretaria de la AMSM; Itzhak Levav es Asesor OMS, Asesor del Ministerio de Salud de Israel; Manuel Desviat, psiquiatra, ha sido Presidente de la AEN; Benedetto Saraceno es Director del Departamento de Salud Mental y Toxicomanías de la Organización Mundial de la Salud, Ginebra. 